\title{
Drug eluting and bare metal stents in people with and without diabetes: collaborative network meta-analysis
}

\begin{abstract}
Christoph Stettler, senior research fellow, ${ }^{1,2,3}$ Sabin Allemann, research fellow, ${ }^{1,2}$ Simon Wandel, research fellow, ${ }^{1}$ Adnan Kastrati, professor of cardiology, ${ }^{4}$ Marie Claude Morice, professor of cardiology, ${ }^{5}$ Albert Schömig, professor of medicine, ${ }^{4}$ Matthias E Pfisterer, professor of cardiology, ${ }^{6}$ Gregg W Stone, professor of medicine, ${ }^{7}$ Martin B Leon, professor of medicine ${ }^{7}$ José Suárez de Lezo, professor of cardiology, ${ }^{8}$ JeanJacques Goy, professor of interventional cardiology, ${ }^{9}$ Seung-Jung Park, professor of cardiology, ${ }^{10}$ Manel Sabaté, associate professor of cardiology, ${ }^{11}$ Maarten I Suttorp, head of department , ${ }^{12}$ Henning Kelbaek, associate professor of cardiology , ${ }^{13}$ Christian Spaulding, professor of cardiology, , ${ }^{14}$ Maurizio Menichelli, interventional cardiologist, ${ }^{15}$ Paul Vermeersch, interventional cardiologist, ${ }^{16}$ Maurits T Dirksen, training fellow in cardiology, ${ }^{17}$ Pavel Cervinka, cardiologist, ${ }^{18}$ Marco De Carlo, vice director, ${ }^{19}$ Andrejs Erglis, associate professor of cardiology, ${ }^{20}$ Tania Chechi, interventional cardiologist, ${ }^{21}$ Paolo Ortolani, interventional cardiologist, ${ }^{22}$ Martin J Schalij, professor of cardiology, ${ }^{23}$ Peter Diem, head of division , ${ }^{2}$ Bernhard Meier, professor of cardiology, ${ }^{24}$ Stephan Windecker, head of invasive cardiology, ${ }^{24,25}$ Peter Jüni, head of division ${ }^{1,25}$
\end{abstract}

\section{${ }^{1}$ Institute of Social and Preventive Medicine, University of Bern, 3012 Bern, Switzerland \\ ${ }^{2}$ Division of Endocrinology, Diabetes and Clinical Nutrition, University Hospital, Bern, Switzerland \\ ${ }^{3}$ International Centre for Circulatory Health, National Heart and Lung Institute, Imperial College, London}

${ }^{4}$ Deutsches Herzzentrum, Technische Universität, Munich, Germany

${ }^{5}$ Institut Hospitalier Jacques Cartier, Massy, France

${ }^{6}$ Division of Cardiology, University Hospital, Basel, Switzerland

${ }^{7}$ Columbia University Medical Center, New York, USA

${ }^{8}$ Servicio de Cardiología, Hospital Universitario Reina Sofía

Cordoba, Spain

${ }^{9}$ Service of Cardiology, Clinique Cecil, Lausanne, Switzerland

${ }^{10}$ Department of Medicine, ASAN Medical Center, Seoul, Korea

${ }^{11}$ Department of Interventional Cardiology, Hospital de San Pau, Barcelona, Spain

${ }^{12}$ Department of Interventional Cardiology, St Antonius Hospital, Nieuwegein, Netherlands

${ }^{13}$ Cardiac Catheterisation Laboratory, Rigshospitalet, Copenhagen, Denmark

${ }^{14}$ Cochin Hospital, Assistance Publique Hôpitaux de Paris, Paris

\section{ABSTRACT}

Objective To compare the effectiveness and safety of three types of stents (sirolimus eluting, paclitaxel eluting, and bare metal) in people with and without diabetes mellitus. Design Collaborative network meta-analysis.

Data sources Electronic databases (Medline, Embase, the Cochrane Central Register of Controlled Trials), relevant websites, reference lists, conference abstracts, reviews, book chapters, and proceedings of advisory panels for the US Food and Drug Administration. Manufacturers and trialists provided additional data.

Review methods Network meta-analysis with a mixed treatment comparison method to combine direct within trial comparisons between stents with indirect evidence from other trials while maintaining randomisation. Overall mortality was the primary safety end point, target lesion revascularisation the effectiveness end point.

Results 35 trials in 3852 people with diabetes and 10947 people without diabetes contributed to the analyses. Inconsistency of the network was substantial for overall mortality in people with diabetes and seemed to be related to the duration of dual antiplatelet therapy ( $P$ value for interaction 0.02). Restricting the analysis to trials with a duration of dual antiplatelet therapy of six months or more, inconsistency was reduced considerably and hazard ratios for overall mortality were near one for all comparisons in people with diabetes: sirolimus eluting stents compared with bare metal stents 0.88 (95\% credibility interval 0.55 to 1.30 ), paclitaxel eluting stents compared with bare metal stents 0.91 (0.60 to 1.38), and sirolimus eluting stents compared with paclitaxel eluting stents 0.95 (0.63 to 1.43). In people without diabetes, hazard ratios were unaffected by the restriction. Both drug eluting stents were associated with a decrease in revascularisation rates compared with bare metal stents in people both with and without diabetes.

Conclusion In trials that specified a duration of dual antiplatelet therapy of six months or more after stent implantation, drug eluting stents seemed safe and effective in people both with and without diabetes.

\section{INTRODUCTION}

People with diabetes experience a more generalised form of atherosclerosis than people without diabetes. They are at an increased risk for coronary heart disease and have more restenoses after the implantation of coronary stents. On average sirolimus eluting stents and paclitaxel eluting stents are associated with a noticeable reduction in target lesion revascularisation compared with bare metal stents, whereas the rates of overall mortality and cardiac mortality associated with the three stents are similar. ${ }^{1}$ Differences in the process and dynamics of restenosis along with variations in metabolic profiles may, however, alter safety or effectiveness profiles of the different stent types, particularly in people with diabetes.

Randomised trials have reported a reduced revascularisation rate with both sirolimus eluting stents and paclitaxel eluting stents compared with bare metal stents in people with diabetes, ${ }^{2-4}$ w1-w3 but the trials were hampered by small numbers of patients and a limited duration of follow-up. A meta-analysis of four early trials in 428 people with diabetes that compared sirolimus eluting stents with bare metal stents for up to four years suggested a strongly increased risk of mortality (hazard ratio 2.90, 95\% confidence interval 
Descartes University, and INSERM U 780, Paris, France

${ }^{15}$ San Camillo Hospital, Rome,

Italy

${ }^{16}$ Antwerp Cardiovascular Institute Middelheim, Antwerp, Belgium

${ }^{17}$ Amsterdam Department of Interventional Cardiology, Onze Lieve Vrouwe Gasthuis,

Amsterdam, Netherlands

${ }^{18}$ Division of Cardiology, Masaryk Hospital, Ústí nad Labem, Czech Republic

${ }^{19}$ Cardiothoracic and Vascular Department, University of Pisa, Italy

${ }^{20}$ University of Latvia, Institute of Cardiology, Riga, Latvia

${ }^{21}$ Cardiologia e Cardiologia Invasiva 2-AOU-Careggi, Florence Italy

${ }^{22}$ Institute of Cardiology, S OrsolaMalpighi Hospital, University of

Bologna, Italy

${ }^{23}$ Department of Cardiology, Leiden University Medical Center Leiden, Netherlands

${ }^{24}$ Department of Cardiology, Bern University Hospital, Bern, Switzerland

${ }^{25}$ CTU Bern, Bern University Hospital, 3010 Bern, Switzerland Correspondence to: $\mathrm{P}$ Jüni juni@ispm.unibe.ch

Cite this as: $B M J$ 2008;337:a1331
1.38 to 6.10$).{ }^{5}$ In view of an average rate of overall mortality of $12 \%$ over four years observed in people with diabetes, ${ }^{1}$ this hazard ratio would translate into a number needed to harm to cause one death over four years as low as 4 (95\% confidence interval 2 to 22$)$. Another meta-analysis, published simultaneously, ${ }^{6}$ also included more recent trials with shorter durations of follow-up: pooling 14 trials in 1411 people with diabetes resulted in a hazard ratio of only 1.27 , but the $95 \%$ confidence interval ranged from 0.83 to 1.95 and was compatible with both moderate benefit and substantial harm.

Network meta-analyses ${ }^{78}$ or mixed treatment comparisons $^{9-11}$ allow a unified, coherent analysis of all randomised controlled trials that compared either of the two drug eluting stents with bare metal stents or the two drug eluting stents head to head, while fully respecting randomisation. In a previous network metaanalysis $^{1}$ we determined the average benefits and harms of all three stent types and provided preliminary results for overall mortality and the composite of death or myocardial infarction stratified according to the presence or absence of diabetes. Here we extend the network meta-analysis stratified according to diabetes status to include 35 trials in 14799 patients, with data from five additional trials, ${ }^{\text {w-w8 }}$ a longer follow-up in one trial, ${ }^{\mathrm{w} 7}$ and data on cardiac death, myocardial infarction, stent thrombosis, and target lesion revascularisation as additional clinical outcomes. ${ }^{12}{ }^{13}$ To address earlier concerns ${ }^{5}$ we prespecified overall mortality as the primary safety outcome and systematically explored the consistency of mortality data in people with diabetes.

\section{METHODS}

We included randomised controlled trials in people with symptoms or signs of myocardial ischaemia as a result of coronary artery disease, that compared the two first generation drug eluting stents approved by the US Food and Drug Administration, a paclitaxel eluting stent (Taxus; Boston Scientific, Natick, MA) and a sirolimus eluting stent (Cypher; Cordis, Miami Lakes, FL) with each other or with a bare metal stent. Trials had to have a clinical follow-up duration of at least six months.

We searched Medline, Embase, the Cochrane Central Register of Controlled Trials (from inception of each database to October 2007), and relevant websites (www.acc.org, www.tctmd.com, www.the heart.org, www.clinicaltrialresults.org) for studies in any language. We checked reference lists, conference abstracts, relevant reviews, book chapters, and the proceedings of the relevant advisory panels of the Food and Drug Administration, and we contacted manufacturers and trialists. (See web extra appendix 1 for details of the search strategy.)

\section{Data extraction}

Two investigators (CSt, SA) extracted data independently, with disagreements resolved in consultation with a third investigator $(\mathrm{PJ})$. We asked the trialists and manufacturers of drug eluting stents to check the extracted information and to provide additional outcome data on an electronic form according to standardised definitions and attempted to obtain outcome data separately for people with and without diabetes.

We specified overall mortality as the primary safety outcome and target lesion revascularisation as the primary effectiveness outcome. Target lesion revascularisation was defined as repeat percutaneous intervention of the target lesion or bypass surgery of the target vessel done for restenosis or other complications of the target lesion (ranging from $5 \mathrm{~mm}$ proximal to $5 \mathrm{~mm}$ distal to the stent). We recorded the following secondary safety outcomes: cardiac death, defined as any death due to a cardiac cause (for example, myocardial infarction, low output failure, fatal arrhyth mia), procedure related deaths, deaths related to concomitant treatment, and death of unknown cause; myocardial infarction, including fatal and non-fatal non- $Q$ wave or $Q$ wave myocardial infarction; a composite of death or myocardial infarction; and stent thrombosis, within the stented segment, confirmed by angiography or post-mortem examination in accordance with the criteria of the Academic Research Consortium, to ensure the inclusion of "secondary" stent thrombosis occurring after a patient had undergone a target lesion revascularisation. In addition, we recorded stent thrombosis according to the definitions used in individual trials (per protocol definition). The numbers of patients experiencing an event and the overall number of patients at risk were recorded separately for years 1 to 4 .

To tackle potential sources of inconsistency in the network we determined the month of completion of patient recruitment as a measure of the period when a trial was done, the duration of follow-up in years, and the duration in months of dual antiplatelet therapy after stent implantation and assessed three key domains of internal validity ${ }^{14}$ : concealment of allocation, blinding of research staff adjudicating clinical outcomes, and the inclusion of all randomised participants in the analysis according to the intention to treat principle. We considered trials to be of high quality that satisfied all three criteria (see also the criteria for quality assessment in the web extra, appendix 1). In most trials the precise durations of dual antiplatelet therapy in individual patients were unavailable. Therefore we obtained all relevant auxiliary information available for trials with a duration of dual antiplatelet therapy of less than six months, such as the information stated in the protocol and the percentage of patients reporting the use of dual antiplatelet therapy at relevant time points. On the basis of this information two investigators (CSt, PJ) independently classified trials according to the likely percentage of patients taking clopidogrel at six months, in increments of $10 \%(<10 \%, 10 \%$ to $<20 \%$, and so on).

\section{Statistical analysis}

We used a hierarchical random effects model ${ }^{15}$ for mixed treatment comparisons ${ }^{9}$ based on piecewise 
constant hazards, with random effects at the levels of trials, adjacent time periods, and comparisons. We simultaneously estimated log hazard ratios for people with and without diabetes and the difference in log hazard ratios between such people. From the posterior distribution of the difference we estimated the $\mathrm{P}$ values for interaction between treatment effect and diabetes status. We excluded from the analyses time periods with zero events in both groups. Hazard ratios were estimated from the median, and the accompanying $95 \%$ credibility intervals from the 2.5th and 97.5th centiles of the posterior distribution. Because of low event rates we derived relative risks of stent thromboses using a previously described random effects Poisson regression model. ${ }^{11}$ As in the preceding study, ${ }^{1}$ we did separate analyses according to time of occurrence of stent thrombosis and an analysis of per protocol definitions of stent thrombosis as used in individual trials (see web extra appendix 1 for details of the models).

We evaluated the inconsistency of the network, defined as the variability of results across different comparisons of the network, by calculating inconsistency factors: the estimated difference between the log hazard ratios from direct comparisons within randomised trials and the log hazard ratios from indirect comparisons between randomised trials with one intervention in common. ${ }^{1}$ To allow intuitive interpretation we back transformed absolute values of these inconsistency factors to ratios of hazard ratios and expressed inconsistency as percentage difference in hazard ratios between direct randomised comparisons within trials and indirect comparisons between trials. Values can range from $0 \%$ to infinity. A value near 0 indicates that all the comparisons in the network are consistent, showing fully coherent estimates of hazard ratios comparing any two types of stent. The more the value deviates from $0 \%$ the more inconsistent the network. A value of $25 \%$, corresponding to a ratio of hazard ratios of 1.25, may be interpreted to indicate low inconsistency, a value of $50 \%$ moderate inconsistency, and a value of $100 \%$, corresponding to a ratio of hazard ratios of 2.00, high inconsistency (see web extra appendix 1). We evaluated heterogeneity between trials, defined as variability of results across trials within comparisons over and above chance, and the goodness of fit of the model to the data (see web extra appendix 1).

To investigate potential sources of variation in the network, we included the characteristics of the trials as covariates in the network meta-analysis of the primary safety outcome. We used prespecified cut-off points of two years for the length of follow-up, January 2004 for completion of patient recruitment, and six months for the duration of dual antiplatelet therapy. In some instances the numbers of trials and events were too low to allow the estimation of random effects at the level of time periods. Therefore we used a random effects Poisson regression model (see web extra appendix 1) for all of these analyses. ${ }^{11} \mathrm{P}$ values for interaction between trial characteristics and treatment effect were derived from the posterior distribution of covariates. The duration of dual antiplatelet therapy specified in trial protocols was the only variable with a treatment by trial characteristic interaction at $\mathrm{P}<0.05$. Therefore we restricted the dataset to trials with a duration of dual antiplatelet therapy of six months or longer and repeated all analyses. Heterogeneity between trials, defined as variability of results across trials within comparisons over and above chance, and the goodness of fit of the model to the data, were evaluated as previously reported (see web extra appendix 1). ${ }^{1}$ All analyses were done in WinBUGS version 1.4.1 and Stata version 9.2 .

\section{RESULTS}

Forty two trials ${ }^{\mathrm{w1}-\mathrm{w} 42}$ met the inclusion criteria (fig 1). Seven trials, totalling about 900 people with diabetes and 3000 without, were excluded because data stratified by diabetes status were not obtainable. ${ }^{\text {w15 }}$ w34 w36 w37 w39 w41 w42 The remaining 35 trials $^{\text {w1-w14 w16-w33 }}$ w35 w38 w40 were included. Investigators or manufacturers provided additional data for 32 trials. ${ }^{\text {w1 w4-w14 w16- }}$ w19 w21-w33 w35 w38 w40 (The characteristics of the 35 included trials are presented in web extra table A.) Four trials ${ }^{\text {w1-w3 }}$ w29 included only people with diabetes and one trial only people without diabetes. ${ }^{\text {w33 }}$ The trials had randomly allocated 3852 people with diabetes mellitus and 10947 people without diabetes mellitus. Patient recruitment started between August 2000 ${ }^{\text {w16 }}$ and October $2004^{\mathrm{w} 4}$ and was completed between January $2001^{\mathrm{w} 16}$ and November 2005. ${ }^{\mathrm{w} 5}$ Data stratified according to the presence or absence of diabetes were available for all 35 trials on all outcomes, except stent thrombosis (see web extra tables B and C). Twenty nine trials described appropriate methods for allocation

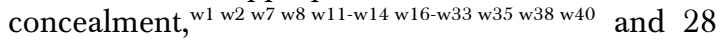
trials reported blind adjudication for clinical out-

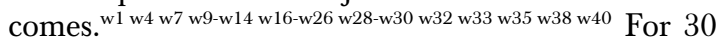
trials all randomised patients could be included in the analyses according to the intention to treat principle. ${ }^{\mathrm{w} 1}$ w3-w7 w9 w11 w13 w14 w16-w23 w25-w33 w35 w38 w40 Twenty four trials were considered to be of high quality. ${ }^{\mathrm{w} 1 \mathrm{w} 7 \mathrm{w} 11 \mathrm{w13}}$ w14 w16-w23 w25 w26 w28-w30 w32 w33 w35 w38 w40

\section{Duration of dual antiplatelet therapy}

The duration of dual antiplatelet therapy specified in the study protocols was two months in five trials, ${ }^{\text {w1 }}{ }^{\text {w18- }}$ w20 w26 three months in three, ${ }^{\text {w3 w7 w17 }}$ six months in $18,{ }^{\text {w2 }}$ w5 w9-w14 w21 w22 w24 w28 w29 w32 w33 w35 w38 w40 nine months in one, ${ }^{\mathrm{w} 4}$ and 12 months in eight. ${ }^{\mathrm{w} 1 \mathrm{w} 6 \mathrm{w} 8 \mathrm{w} 23 \mathrm{w} 25 \text { w27 w30 w31 }}$ All eight trials with therapy lasting less than six months compared sirolimus eluting stents with bare metal stents. Auxiliary information for these trials (see web extra appendix 2) indicated that the percentage of patients actually receiving dual antiplatelet therapy of six months or more was likely to be below $10 \%$ in five trials. ${ }^{\mathrm{w} 7 \mathrm{w} 16-\mathrm{w} 19}$ The likely percentage for the other three trials was between $10 \%$ and $20 \%$, ${ }^{\text {w20 }} 40 \%$ and $50 \%$, ${ }^{\text {w26 }}$ and $50 \%$ and $60 \% .{ }^{\text {w3 }}$ One head to head comparison had specified a minimal duration of dual antiplatelet therapy of two months for sirolimus eluting stents 


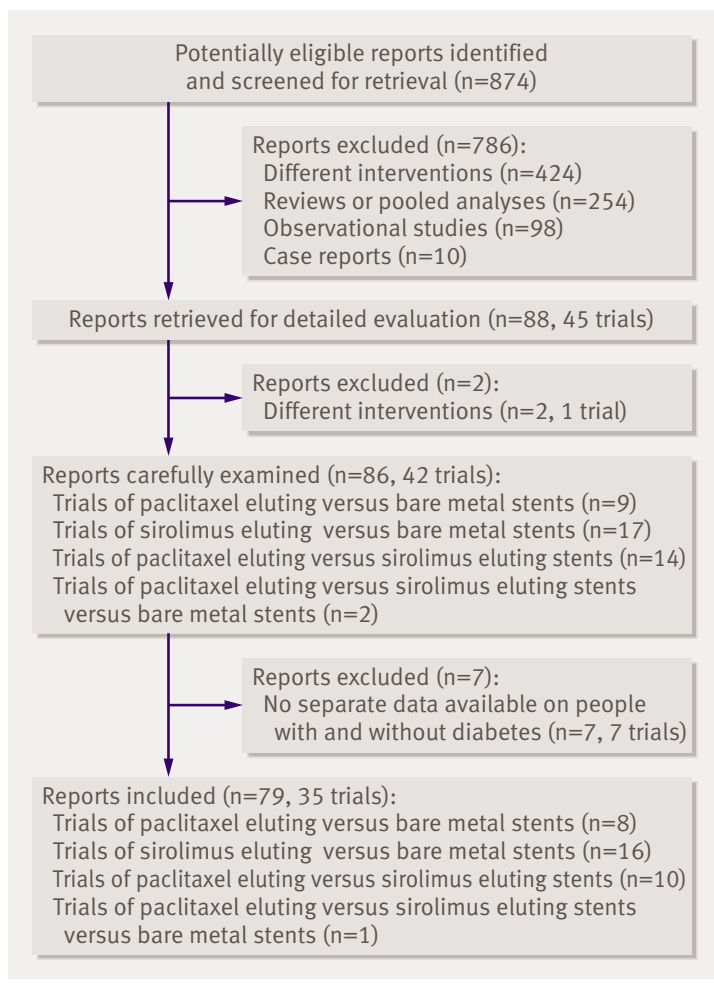

Fig 1| Identification of trials

and six months for paclitaxel eluting stents in the protocol, but the actual duration was only one month shorter for patients allocated to siroliumus stents than for patients allocated to paclitaxel stents, and about $50 \%$ of patients allocated to either stent type were still receiving therapy after eight months. ${ }^{\text {w32 }}$

Network of all trials: overall mortality

Table 1 presents the results of the network metaanalysis of overall mortality in people with and without diabetes. In people with diabetes the estimated hazard ratio for sirolimus eluting compared with bare metal stents was 1.14 (95\% credibility interval 0.74 to 1.60$)$, for paclitaxel eluting versus bare metal stents was 1.09 (0.71 to 1.66), and for sirolimus versus paclitaxel eluting stents was 1.02 (0.70 to 1.57), and compatible with both a substantial harm and a moderate benefit of either eluting stent compared with bare metal stents. The corresponding values for people without diabetes were 1.02 (0.77 to 1.29 ), 0.90 (0.67 to 1.16 ), and 1.13 (0.83 to 1.54 ; table 1$)$. A moderate to high inconsistency of $61 \%$ was found among people with diabetes, but none among people without diabetes.

\section{Exploration of sources of variation}

Table 2 presents an investigation into potential sources of variation in people with diabetes in the network. Estimates of relative risk comparing sirolimus eluting stents with paclitaxel eluting stents depended to some extent on the quality of the trials, the length of followup, and the time of completion of patient recruitment (table 2), but $95 \%$ credibility intervals were wide and tests for interaction negative ( $\mathrm{P}$ for interaction $\geq 0.16$ ). The estimated relative risk of death when sirolimus eluting stents were compared with bare metal stents was greater when the specified duration of dual antiplatelet therapy was less than six months $(2.37$, $95 \%$ credibility interval 1.18 to 5.12 ) compared with six months or longer $(0.89,0.58$ to $1.40, \mathrm{P}$ for interaction $0.02)$, however. When three trials originally classified to have a short duration of dual antiplatelet therapy ${ }^{\mathrm{w} 3}$ ${ }^{\text {w20 }}$ w26 were reclassified to have a duration of six months or longer, reflecting the auxiliary information indicating that more than $10 \%$ of patients in these trials were still receiving dual antiplatelet therapy at six months, differences were maintained.

\section{Restricted network: overall mortality}

When the network was restricted to trials with dual antiplatelet therapy for six months or longer (table 1), the hazard ratios of death overall among people with diabetes were all below 1: sirolimus eluting stents compared with bare metal stents 0.88 (95\% credibility interval 0.55 to 1.30 ), paclitaxel eluting stents compared with bare metal stents 0.91 (0.60 to 1.38), and sirolimus eluting compared with paclitaxel eluting stents 0.95 (0.63 to 1.43). Compared with the network of all trials the inconsistency decreased to $20 \%$ and credibility intervals of hazard ratios became more narrow in the restricted network. Among patients without diabetes results were much the same in the overall and the restricted network (table 1). The hazard ratio for sirolimus eluting stents compared with bare metal stents was 1.05 (0.69 to 1.73), for paclitaxel eluting stents compared with bare metal stents it was 0.89 (0.66 to 1.18), and for sirolimus compared with paclitaxel eluting stents it was 1.23 (0.82 to 1.69). Figure 2 presents corresponding cumulative incidences of death for the three stent types estimated from the restricted network meta-analysis separately for people with and without diabetes. The incidence of death was about twice as high in people with diabetes compared with people without diabetes. Tests for interaction between treatment effect and diabetes status were negative for all comparisons ( $\mathrm{P}$ for interaction $\geq 0.28$; also see web extra table D).

\section{Restricted network: secondary safety outcomes}

Table 1 allows a comparison of the results from the network meta-analysis of all trials and the analysis restricted to trials with a dual antiplatelet therapy of six months or more. Among people with diabetes, hazard ratios for drug eluting stents compared with bare metal stents became more beneficial for drug eluting stents for the outcomes of cardiac death, the composite of death or myocardial infarction, and for stent thromboses. The inconsistency decreased mainly for cardiac death and per protocol definitions of stent thromboses. No differences between overall and restricted network meta-analysis were observed for myocardial infarction. Among people without diabetes, results from overall and restricted network meta-analysis were similar. Corresponding cumulative incidences for the three 
Table 1| Analyses overall and restricted to trials with dual antiplatelet therapy of at least six months

\begin{tabular}{|c|c|c|c|c|c|c|c|c|}
\hline \multirow[b]{3}{*}{ Variable and stent types } & \multicolumn{4}{|c|}{ People with diabetes } & \multicolumn{4}{|c|}{ People without diabetes } \\
\hline & \multicolumn{2}{|l|}{ All trials } & \multicolumn{2}{|c|}{$\begin{array}{l}\text { Trials with dual antiplatelet } \\
\text { therapy } \geq 6 \text { months }\end{array}$} & \multicolumn{2}{|l|}{ All trials } & \multicolumn{2}{|c|}{$\begin{array}{l}\text { Trials with dual antiplatelet } \\
\text { therapy } \geq 6 \text { months }\end{array}$} \\
\hline & Hazard ratio $(95 \% \mathrm{Cl})$ & IC* & Hazard ratio $(95 \% \mathrm{Cl})$ & $I C^{\star}$ & Hazard ratio $(95 \% \mathrm{Cl})$ & $I C^{*}$ & Hazard ratio $(95 \% \mathrm{Cl})$ & $I^{*}$ \\
\hline \multicolumn{9}{|l|}{ Death overall: } \\
\hline SES $v$ bare metal stent & $1.14(0.74$ to 1.60$)$ & \multirow{3}{*}{$61 \%$} & $0.88(0.55$ to 1.30$)$ & \multirow{3}{*}{$20 \%$} & $1.02(0.77$ to 1.29$)$ & \multirow{3}{*}{$3 \%$} & $1.05(0.69$ to 1.73$)$ & \multirow{3}{*}{$1 \%$} \\
\hline PES $v$ bare metal stent & $1.09(0.71$ to 1.66$)$ & & 0.91 (0.60 to 1.38$)$ & & $0.90(0.67$ to 1.16$)$ & & $0.89(0.66$ to 1.18$)$ & \\
\hline SES $v$ PES & $1.02(0.70$ to 1.57$)$ & & 0.95 (0.63 to 1.43$)$ & & $1.13(0.83$ to 1.54$)$ & & $1.23(0.82$ to 1.69$)$ & \\
\hline \multicolumn{9}{|l|}{ Cardiac death: } \\
\hline SES $v$ bare metal stent & 1.09 (0.63 to 1.93$)$ & \multirow{3}{*}{$24 \%$} & $0.80(0.42$ to 1.57$)$ & \multirow{3}{*}{$4 \%$} & 0.88 (0.48 to 1.60$)$ & \multirow{3}{*}{$10 \%$} & $0.93(0.45$ to 1.81$)$ & \multirow{3}{*}{$12 \%$} \\
\hline PES $v$ bare metal stent & $1.08(0.62$ to 2.28$)$ & & 0.94 (0.52 to 1.87$)$ & & $0.90(0.52$ to 1.54$)$ & & $0.89(0.55$ to 1.47$)$ & \\
\hline SES $v$ PES & 0.98 (0.46 to 2.08$)$ & & $0.85(0.50$ to 1.55$)$ & & $0.96(0.58$ to 1.84$)$ & & $1.04(0.54$ to 1.95$)$ & \\
\hline \multicolumn{9}{|l|}{ Myocardial infarction: } \\
\hline SES $v$ bare metal stent & $0.68(0.44$ to 1.05$)$ & \multirow{3}{*}{$30 \%$} & $0.68(0.43$ to 1.12$)$ & \multirow{3}{*}{$30 \%$} & 0.87 (0.64 to 1.20$)$ & \multirow{3}{*}{$5 \%$} & $0.81(0.55$ to 1.14$)$ & \multirow{3}{*}{$20 \%$} \\
\hline PES $v$ bare metal stent & $0.84(0.55$ to 1.31$)$ & & $0.85(0.54$ to 1.43$)$ & & $1.08(0.74$ to 1.51$)$ & & $1.05(0.72$ to 1.42$)$ & \\
\hline SES $v$ PES & 0.79 (0.56 to 1.23$)$ & & 0.80 (0.55 to 1.27$)$ & & 0.81 (0.58 to 1.06$)$ & & 0.75 (0.57 to 1.07$)$ & \\
\hline
\end{tabular}

Death or myocardial

infarction:

\begin{tabular}{|c|c|c|c|c|c|c|c|c|}
\hline SES $v$ bare metal stent & 1.04 (0.75 to 1.61$)$ & \multirow{3}{*}{$57 \%$} & 0.88 (0.57 to 1.27$)$ & \multirow{3}{*}{$56 \%$} & $0.93(0.71$ to 1.13$)$ & \multirow{3}{*}{$6 \%$} & 0.91 (0.69 to 1.13$)$ & \multirow{3}{*}{$13 \%$} \\
\hline PES $v$ bare metal stent & 1.07 (0.74 to 1.62$)$ & & 0.91 (0.70 to 1.31$)$ & & $1.04(0.84$ to 1.31$)$ & & $1.00(0.84$ to 1.25$)$ & \\
\hline SES $v$ PES & $0.97(0.72$ to 1.34$)$ & & 0.95 (0.69 to 1.27$)$ & & $0.90(0.71$ to 1.09$)$ & & $0.88(0.71$ to 1.06$)$ & \\
\hline
\end{tabular}

Stent thrombosis (ARC

definite):

\begin{tabular}{|c|c|c|c|c|c|c|c|c|}
\hline SES $v$ bare metal stent & $0.46(0.15$ to 1.42$)$ & \multirow{3}{*}{$26 \%$} & 0.33 (0.09 to 1.09$)$ & \multirow{3}{*}{$24 \%$} & 1.35 (0.76 to 2.73$)$ & \multirow{3}{*}{$32 \%$} & 1.24 (0.58 to 3.08$)$ & \multirow{3}{*}{$20 \%$} \\
\hline PES $v$ bare metal stent & $1.05(0.32$ to 4.01$)$ & & 0.82 (0.23 to 3.09$)$ & & 1.54 (0.83 to 3.13$)$ & & 1.48 (0.69 to 3.40$)$ & \\
\hline SES $v$ PES & $0.44(0.15$ to 1.17$)$ & & $0.40(0.13$ to 1.08$)$ & & 0.87 (0.47 to 1.69$)$ & & 0.84 (0.41 to 1.88$)$ & \\
\hline
\end{tabular}

\section{Stent thrombosis (per}

protocol):

\begin{tabular}{|c|c|c|c|c|c|c|c|c|}
\hline SES $v$ bare metal stent & $0.48(0.17$ to 1.35$)$ & \multirow{3}{*}{$20 \%$} & $0.20(0.05$ to 0.68$)$ & \multirow{3}{*}{$5 \%$} & $1.43(0.78$ to 3.00$)$ & \multirow{3}{*}{$8 \%$} & $1.48(0.74$ to 3.41$)$ & \multirow{3}{*}{$10 \%$} \\
\hline PES $v$ bare metal stent & $1.27(0.38$ to 4.91$)$ & & $0.73(0.19$ to 2.80$)$ & & $1.73(0.88$ to 3.61$)$ & & $1.80(0.89$ to 3.67$)$ & \\
\hline SES $v$ PES & 0.38 (0.11 to 1.07$)$ & & $0.27(0.07$ to 0.80$)$ & & $0.82(0.44$ to 1.73$)$ & & $0.82(0.44$ to 1.73$)$ & \\
\hline \multicolumn{9}{|l|}{$\begin{array}{l}\text { Target lesion } \\
\text { revascularisation: }\end{array}$} \\
\hline SES $v$ bare metal stent & $0.29(0.22$ to 0.39$)$ & \multirow{3}{*}{$2 \%$} & $0.29(0.19$ to 0.45$)$ & \multirow{3}{*}{$0 \%$} & $0.29(0.22$ to 0.38$)$ & \multirow{3}{*}{$3 \%$} & 0.29 (0.19 to 0.42$)$ & \multirow{3}{*}{$1 \%$} \\
\hline PES $v$ bare metal stent & $0.38(0.28$ to 0.55$)$ & & $0.38(0.26$ to 0.56$)$ & & $0.46(0.33$ to 0.60$)$ & & $0.46(0.32$ to 0.60$)$ & \\
\hline SES $v$ PES & $0.76(0.53$ to 1.05$)$ & & $0.78(0.50$ to 1.14$)$ & & $0.63(0.49$ to 0.82$)$ & & 0.64 (0.49 to 0.84$)$ & \\
\hline
\end{tabular}

SES=sirolimus eluting stent; PES=paclitaxel eluting stent; $A R C=A c a d e m i c ~ R e s e a r c h$ Consortium.

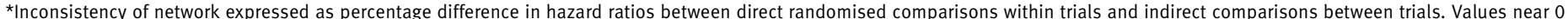

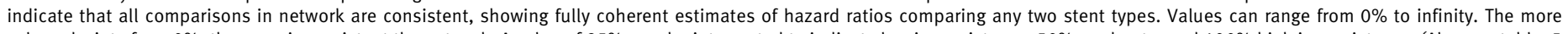

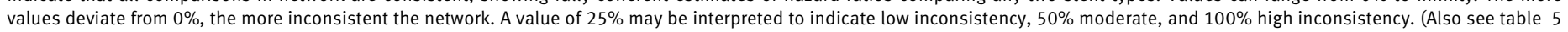
in web extra appendix 3 for $95 \%$ credibility intervals and P values of inconsistency estimates.)

stent types are presented in figures 2 and 3. Again, incidences were higher in people with diabetes than without, with most pronounced differences observed for cardiac death. Tests for interaction between treatment effect and diabetes status were negative for all comparisons on cardiac death, myocardial infarction, and their composite ( $\mathrm{P}$ for interaction $\geq 0.47$, see also web extra table D).

Table 3 presents a breakdown of stent thromboses according to time of occurrence. Among people with diabetes little evidence was found for an increased risk of definite or per protocol stent thrombosis associated with sirolimus eluting stents compared with either of the two other stents; all point estimates were below 1 and differences in favour of sirolimus eluting stents became more pronounced with the use of per protocol definitions. For the comparison of paclitaxel eluting stents with bare metal stents all estimates were imprecise for both the Academic Research
Consortium definition of definite stent thrombosis and per protocol definitions. Among people without diabetes relative risks were generally higher for both definitions, but tests for interaction between treatment effect and diabetes status were positive only for the comparison of sirolimus eluting stents with bare metal stents on per protocol definitions of stent thrombosis between day 0 and 4 years and between day 30 and 4 years $(\mathrm{P}$ for interaction $=0.01$, see also web extra table D).

Restricted network: target lesion revascularisation Both drug eluting stents were robustly associated with a decrease in revascularisation rates compared with bare metal stents, network inconsistency was low, and results were unaffected by the restriction of the analysis to trials with a duration of dual antiplatelet therapy of six months or more in people with and without diabetes (table 1). Differences between sirolimus and paclitaxel 
Table 2 | Overall mortality in patients with diabetes: evaluation of variation in network according to different trial characteristics

\begin{tabular}{|c|c|c|c|c|c|c|}
\hline \multirow[b]{2}{*}{ Characteristic } & \multicolumn{2}{|c|}{ SES $v$ bare metal stent } & \multicolumn{2}{|c|}{ PES $v$ bare metal stent } & \multicolumn{2}{|c|}{ SES $v$ PES } \\
\hline & Relative risk $(95 \% \mathrm{Cl})$ & P value for interaction & Relative risk $(95 \% \mathrm{Cl})$ & $P$ value for interaction & Relative risk $(95 \% \mathrm{Cl})$ & $P$ value for interaction \\
\hline \multicolumn{7}{|l|}{ Concealment of allocation: } \\
\hline Adequate & $1.30(0.86$ to 2.02$)$ & \multirow{2}{*}{0.16} & 1.22 (0.74 to 1.99$)$ & \multirow{2}{*}{0.72} & 1.06 (0.69 to 1.67$)$ & \multirow{2}{*}{-} \\
\hline Unclear & 0.32 (0.03 to 2.27$)$ & & $0.93(0.21$ to 4.33$)$ & & - & \\
\hline \multicolumn{7}{|l|}{ Blind adjudication: } \\
\hline Yes & $1.30(0.84$ to 2.16$)$ & \multirow{2}{*}{0.37} & 1.17 (0.67 to 1.96$)$ & \multirow{2}{*}{0.96} & 1.11 (0.69 to 2.04$)$ & \multirow{2}{*}{0.78} \\
\hline No & $0.72(0.17$ to 2.46$)$ & & $1.24(0.10$ to 11.76$)$ & & 0.94 (0.26 to 2.64$)$ & \\
\hline \multicolumn{7}{|l|}{ Intention to treat analysis: } \\
\hline Yes & 1.25 (0.81 to 2.02$)$ & \multirow{2}{*}{0.71} & 1.13 (0.65 to 1.92$)$ & \multirow{2}{*}{0.92} & 1.11 (0.71 to 1.87$)$ & \multirow{2}{*}{ Not estimable* } \\
\hline No or unclear & 0.97 (0.26 to 3.82) & & $1.08(0.37$ to 3.23$)$ & & $0.14(0.01 \text { to } 3.10)^{\star}$ & \\
\hline \multicolumn{7}{|l|}{ High quality trial: } \\
\hline Yes & $1.40(0.86$ to 2.49$)$ & \multirow{2}{*}{0.27} & $1.28(0.66$ to 2.44$)$ & \multirow{2}{*}{0.61} & $1.08(0.64$ to 2.14$)$ & \multirow{2}{*}{0.80} \\
\hline No & $0.70(0.21$ to 2.18$)$ & & 0.97 (0.37 to 2.52$)$ & & $0.93(0.26$ to 2.77$)$ & \\
\hline \multicolumn{7}{|l|}{ Length of follow-up: } \\
\hline$>2$ years & 1.37 (0.80 to 2.48$)$ & \multirow{2}{*}{0.51} & $1.30(0.71$ to 2.46$)$ & \multirow{2}{*}{0.54} & 1.05 (0.61 to 1.90$)$ & \multirow{2}{*}{0.97} \\
\hline$\leq 2$ years & 1.01 (0.47 to 2.19$)$ & & 0.91 (0.34 to 2.48$)$ & & $1.02(0.29$ to 4.13$)$ & \\
\hline \multicolumn{7}{|l|}{ Patient recruitment: } \\
\hline Completed Jan 2004 or later & $0.93(0.52$ to 1.73$)$ & \multirow{2}{*}{0.93} & 1.11 (0.60 to 2.06$)$ & \multirow{2}{*}{0.54} & 0.83 (0.54 to 1.33$)$ & \multirow{2}{*}{0.94} \\
\hline Completed before Jan 2004 & $1.70(0.96$ to 3.08$)$ & & $0.84(0.41$ to 1.81$)$ & & $3.68(0.41 \text { to } 32.9)^{\star}$ & \\
\hline \multicolumn{7}{|l|}{ Dual antiplatelet therapy: } \\
\hline$\geq 6$ months & $0.89(0.58$ to 1.40$)$ & \multirow{2}{*}{0.02} & 0.97 (0.64 to 1.49$)$ & \multirow{2}{*}{-} & 0.92 (0.64 to 1.34$)$ & \multirow[b]{2}{*}{ 一 } \\
\hline$<6$ months & 2.37 (1.18 to 5.12$)$ & & - & & - & \\
\hline
\end{tabular}

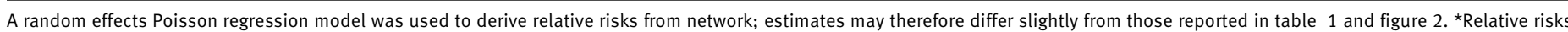
for these comparisons are derived from one trial.

*Single trial had zero events in one arm.

eluting stents tended to be less pronounced among people with diabetes, and the credibility intervals overlapped 1 (fig 4). Tests of interaction between treatment effect and diabetes status were negative $(\mathrm{P}$ values for interaction $\geq 0.44$, see also web extra table $\mathrm{D}$ ).

Between trial heterogeneity, model fit, and comparison with conventional meta-analyses

Web extra appendix 3 presents results from the evaluations of heterogeneity between trials and model fit. After restricting the network to trials with dual antiplatelet therapy of six months or more, heterogeneity between trials was low for all outcomes except stent thrombosis. For people without diabetes heterogeneity between trials was low for death, myocardial infarction, and the composite of death or myocardial infarction, and moderate for most comparisons on the other three outcomes. The model fit was adequate for all outcomes. Web extra table E presents a comparison of results from network meta-analyses with results from conventional meta-analyses: all estimates were compatible.

\section{DISCUSSION}

Our collaborative network meta-analysis suggests that previously reported increases in the risk of death associated with sirolimus eluting stents compared with bare metal stents in people with diabetes ${ }^{5}$ probably resulted from the restricted duration of dual antiplatelet therapy of less than six months in early trials. In trials with dual antiplatelet therapy for less than six months, the risk of death associated with sirolimus eluting stent was more than twice the risk associated with bare metal stents, which translates into a number needed to harm as low as 7 to cause one death over four years. Conversely, trials with dual antiplatelet therapy for six months or more showed no increase in risk from using sirolimus eluting stents compared with bare metal stents. Restricting the network to trials with dual antiplatelet therapy of six months or longer resulted in a clear reduction of the inconsistency and hazard ratios near 1 , which were robust to all sensitivity analyses. We found similar patterns for analyses of cardiac death and stent thromboses. The restriction of the network resulted in a smaller evidence base: eight trials of 613 people with diabetes were excluded from the analysis. Despite this, statistical precision was improved owing to the accompanying decrease in the network's inconsistency.

Compared with bare metal stents, target lesion revascularisation rates are strongly decreased by use of sirolimus and paclitaxel drug eluting stents in people with and without diabetes. Numbers needed to treat to reduce one event over four years are 6 in people with diabetes and 8 in people without diabetes. Active angiographic follow-up increases the absolute rates of target lesion revascularisation. ${ }^{1617}$ For example, the rate of target lesion revascularisation at one year in patients who had undergone implantation of a bare metal stent in the TAXUS IV trial was $18.9 \%$ in those allocated to active follow-up and $14.3 \%$ in those allocated to regular clinical follow-up without 

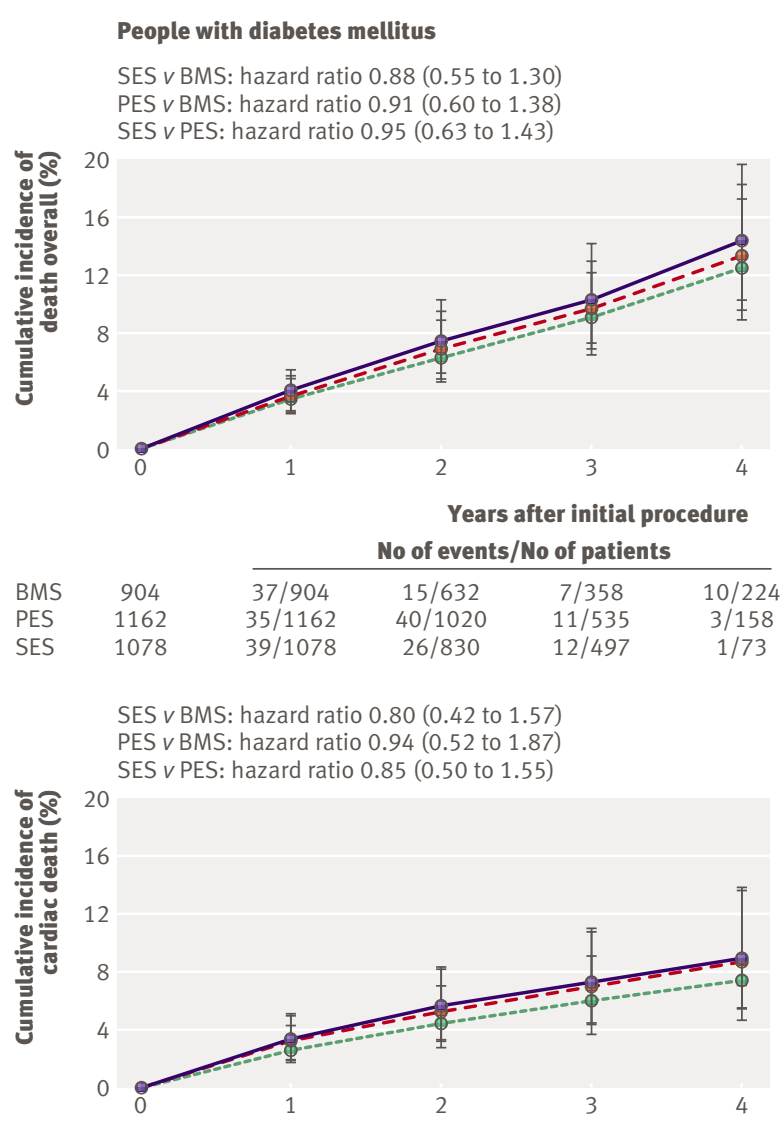

\begin{tabular}{|c|c|c|c|c|c|}
\hline \multirow[b]{2}{*}{ BMS } & \multirow[b]{2}{*}{900} & & \multicolumn{3}{|c|}{$\begin{array}{l}\text { Years after initial procedure } \\
\text { o of events/No of patients }\end{array}$} \\
\hline & & $26 / 900$ & $9 / 628$ & $1 / 354$ & $2 / 22$ \\
\hline PES & 1036 & $27 / 1036$ & $18 / 935$ & $5 / 494$ & $0 / 152$ \\
\hline SES & 962 & $24 / 962$ & $15 / 745$ & $6 / 464$ & $0 / 73$ \\
\hline
\end{tabular}

People without diabetes mellitus

SES $v$ BMS: hazard ratio 1.05 (0.69 to 1.73$)$ PES $v$ BMS: hazard ratio 0.89 (0.66 to 1.18$)$ SES $v$ PES: hazard ratio 1.23 (0.82 to 1.69 )

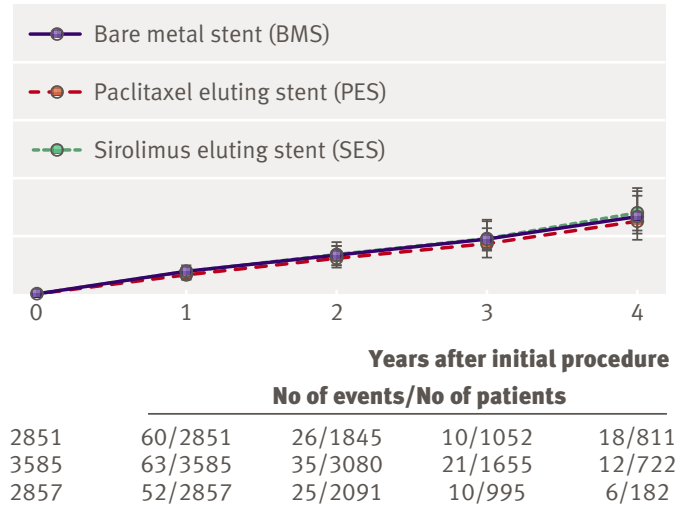

SES $v$ BMS: hazard ratio 0.93 (0.45 to 1.81$)$ PES $v$ BMS: hazard ratio 0.89 (0.55 to 1.47$)$ SES $v$ PES: hazard ratio 1.04 (0.54 to 1.95)

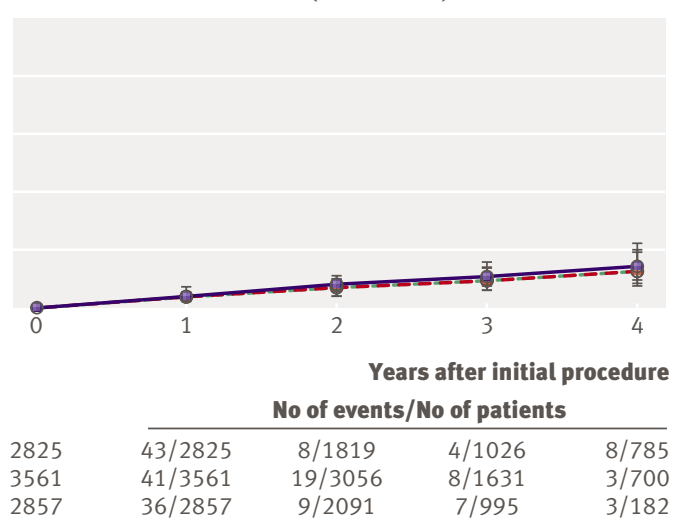

$\overline{\text { Fig } 2}$ | Cumulative incidence of death overall and cardiac death and corresponding hazard ratios (95\% credibility intervals) for three stent types estimated from network meta-analysis for pairwise comparisons in people with and without diabetes and restricted to trials with dual antiplatelet therapy of at least six months

mandatory angiography. The number needed to treat to avoid one revascularisation would therefore be somewhat lower in clinical routine. Assuming revascularisation rates of $12 \%$ in people with diabetes and $9 \%$ in people without diabetes, as found in the Cardiac Care Network of Ontario at two years, ${ }^{18}$ numbers needed to treat were estimated as 13 for people with diabetes and 18 for people without diabetes.

Our study comprises a large body of evidence from randomised controlled trials in people with and without diabetes treated with one of two drug eluting stents or bare metal stents. Investigators and manufacturers provided additional data according to uniform outcome definitions, including a standardised definition for stent thrombosis according to the Academic Research Consortium consensus. ${ }^{13}$ This increases comparability between trials and limits bias, such as the censoring of events after intervening revascularisation.

Our previous network meta-analysis ${ }^{1}$ was recently criticised for integrating evidence from direct and indirect comparisons, for not comparing like with like, and for using random effects models that give undue weight to small studies. ${ }^{19}$ Our model was based on relative treatment effects (log hazards ratio), and variations in characteristics of patients or lesions between trials are fully accounted for in the analysis by maintaining randomised comparisons within each trial. ${ }^{1}$ Network meta-analysis makes similar assumptions to standard meta-analysis of direct comparisons within trials, but requires that these assumptions hold over the entire set of trials in the network. ${ }^{10}$ The smaller the heterogeneity between trials and the smaller the inconsistency of the data, the more likely relative treatment effects originate from the same distribution and less likely small trials get undue weight in the analysis. Therefore a careful exploration of heterogeneity between trials and inconsistency of the data is mandatory. Inspecting the entire network allowed us to gain insights into potential sources of variation, which could not have been achieved by an isolated look at just one set of trials comparing only two stent types. This led us to suggest a plausible explanation for the previously reported increase in the risk of death associated with sirolimus eluting stents compared with bare metal stents in people with diabetes ${ }^{5}$ - that is, a restricted 


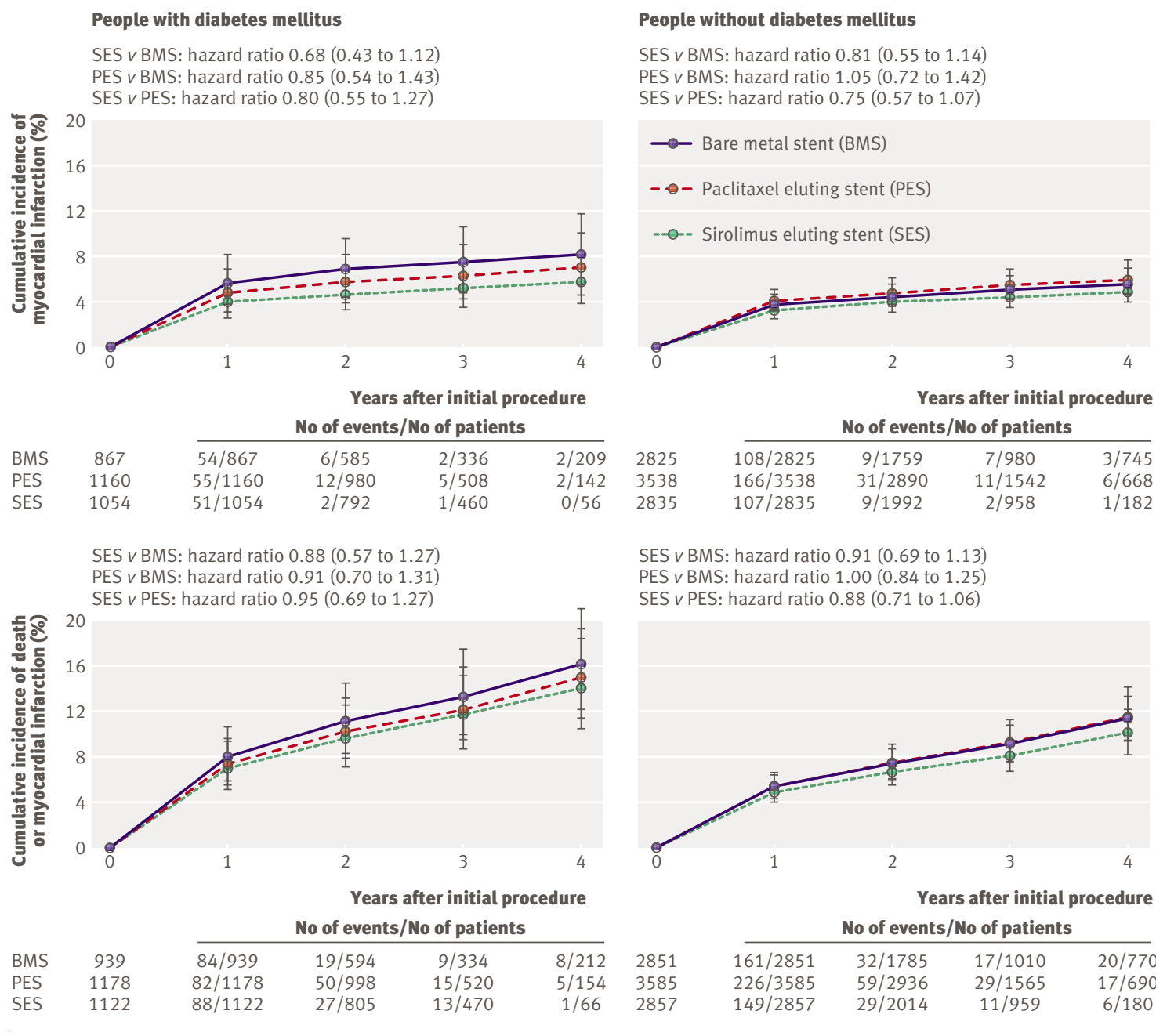

Fig 3 Cumulative incidence of myocardial infarction and the composite of death or myocardial infarction and corresponding hazard ratios ( $95 \%$ credibility intervals) for three stent types estimated from network meta-analysis for pairwise comparisons in people with and without diabetes and restricted to trials with dual antiplatelet therapy of at least six months

duration of dual antiplatelet therapy particularly in early trials.

Our exploration of inconsistency is observational in nature and has the same limitations as other observational studies. ${ }^{20}$ Most importantly, earlier trials had specified shorter durations of dual antiplatelet therapy than later trials. The duration of therapy was therefore bound to be negatively correlated with the duration of follow-up, and confounding could exist between the duration of therapy and the length of follow-up. Other potential confounders include changes over time in patient selection and procedural characteristics, such as an under-sizing or under-expansion of stents in early trials, or methodological quality. We addressed this by repeating tests of interaction between treatment effect and components of methodological quality or length of follow-up after the exclusion of trials with a duration of dual antiplatelet therapy of less than six months and found no evidence for an interaction in any of these analyses (data available on request).

We acknowledge that our results could be corroborated by an analysis of the actual duration of dual antiplatelet therapy in individual patients, but precise durations in individual patients are unavailable in most trials and we lacked the resources to retrospectively ascertain and validate usage data. Eight trials had specified a duration of dual antiplatelet therapy of less than six months. For five trials we are confident that the actual duration of dual antiplatelet therapy corresponded to the specified duration in at least $90 \%$ of patients $^{\text {w7 }}{ }^{\text {w16-w19}}$; in three trials, however, between 10\% and $50 \%$ of the patients had dual antiplatelet therapy at six months. ${ }^{\text {w3 w20 w26 }}$ Results were unaffected by the reclassification of these three trials to have a duration of dual antiplatelet therapy of six months or longer, and the Pvalue for interaction between relative risk of death and duration of dual antiplatelet therapy became even smaller. Additionally, strut thickness or type of bare metal stent used in comparison groups might affect clinical outcomes. ${ }^{\text {w21 }}$ Even though our results are robust to the adjustment for these characteristics of bare metal stents, ${ }^{1}$ we cannot fully exclude the possibility that differences in bare metal stents as comparators contributed to the observed variation in 
Table $3 \mid$ Stent thromboses in trials with dual antiplatelet therapy of six months or more

\begin{tabular}{|c|c|c|c|c|c|c|c|}
\hline \multirow[b]{2}{*}{ Variable } & \multicolumn{4}{|c|}{ Events } & \multicolumn{3}{|c|}{ Relative risks ( $95 \%$ credibility interval) } \\
\hline & BMS & PES & SES & Total & SES $v$ BMS & PES $v$ BMS & SES $v$ PES \\
\hline \multicolumn{8}{|c|}{ ARC definite stent thrombosis* } \\
\hline No of patients at risk & 557 & 874 & 753 & 2184 & & & \\
\hline 0 days to 4 years & 13 & 17 & 9 & 39 & 0.33 (0.09 to 1.09$)$ & 0.82 (0.23 to 3.09$)$ & $0.40(0.13$ to 1.08$)$ \\
\hline$>30$ days to 4 years & 2 & 8 & 3 & 13 & $0.72(0.04$ to 10.8$)$ & $3.54(0.23$ to 78.6$)$ & $0.20(0.02$ to 1.04$)$ \\
\hline \multicolumn{8}{|l|}{ Patients without diabetes: } \\
\hline No of patients at risk & 2439 & 3130 & 2647 & 8216 & & & \\
\hline 0 days to 4 years & 34 & 56 & 46 & 136 & 1.24 (0.58 to 3.08$)$ & $1.48(0.69$ to 3.40$)$ & $0.84(0.41$ to 1.88$)$ \\
\hline \multicolumn{8}{|l|}{ Patients with diabetes: } \\
\hline No of patients at risk & 723 & 912 & 870 & 2505 & & & \\
\hline 0 days to 4 years & 16 & 18 & 7 & 41 & $0.20(0.05$ to 0.68$)$ & $0.73(0.19$ to 2.80$)$ & 0.27 (0.07 to 0.80$)$ \\
\hline $0-30$ days & 11 & 10 & 5 & 26 & 0.23 (0.03 to 1.08$)$ & 0.55 (0.09 to 3.05$)$ & $0.42(0.07$ to 1.89$)$ \\
\hline$>30$ days to 4 years & 5 & 8 & 2 & 15 & $0.10(0.01$ to 0.93$)$ & 0.87 (0.06 to 10.3$)$ & 0.11 (0.01 to 0.75$)$ \\
\hline \multicolumn{8}{|l|}{ Patients without diabetes: } \\
\hline No of patients at risk & 2577 & 3382 & 2625 & 8584 & & & \\
\hline 0 days to 4 years & 29 & 58 & 46 & 133 & $1.48(0.74$ to 3.41$)$ & $1.80(0.89$ to 3.67$)$ & $0.82(0.44$ to 1.73$)$ \\
\hline $0-30$ days & 22 & 24 & 28 & 74 & $1.11(0.47$ to 2.81$)$ & 0.99 (0.44 to 2.33$)$ & 1.15 (0.48 to 2.72$)$ \\
\hline
\end{tabular}

BMS=bare metal stent; $\mathrm{PES}=$ paclitaxel eluting stent; $\mathrm{SES}=$ sirolimus eluting stent.

*According to Academic Research Consortium criteria.

†According to protocol definitions used in individual trials.

mortality between trials with short and long durations of dual antiplatelet therapy. Four trials included only people with diabetes ${ }^{\mathrm{w1}-\mathrm{w} 3}$ w29 and one trial only people without diabetes. ${ }^{\text {w33 }}$ Performing network meta-analyses separately for people with and without diabetes allowed us to also incorporate these trials in our analysis. An alternative approach would have been to model differences between people with and without diabetes directly within each trial, but at the price of excluding these five trials. ${ }^{\text {w-w3 w29 w33 }} \mathrm{A}$ final limitation of our study is that we were unable to record information on specific antidiabetic treatment or on glycaemic control in people with diabetes mellitus and to perform separate analyses for people with diabetes who did or did not use insulin. Although these aspects are related to cardiovascular outcomes, ${ }^{21}$ they were per

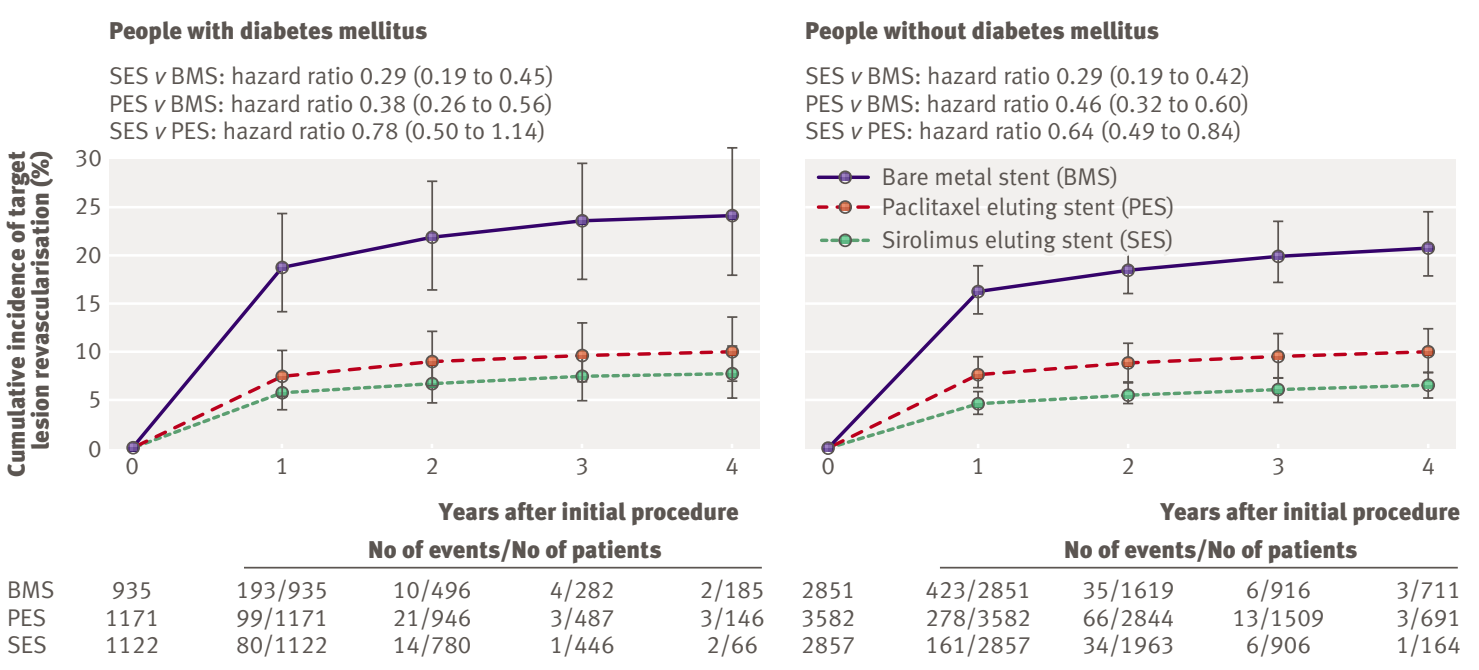

Fig 4 | Cumulative incidence of target lesion revascularisation and corresponding hazard ratios (95\% credibility intervals) for three stent types estimated from network meta-analysis for pairwise comparisons in people with and without diabetes and restricted to trials with dual antiplatelet therapy of at least six months 
definition randomly distributed across comparison groups within each trial and it seems unlikely that they influenced results.

We found that the duration of dual antiplatelet therapy modified the safety profile of drug eluting stents mainly in people with diabetes. The beneficial effect of prolonged therapy in people with diabetes may be mainly related to differences in lesion characteristics. People with diabetes tend to have smaller vessels and longer lesions than people without diabetes and therefore require stents of smaller size and longer length. Previous studies have identified both vessel size and lesion length as predictors of stent thrombosis, ${ }^{22}$ which may explain the predisposition of people with diabetes to this adverse event in the absence of adequate antiplatelet therapy. In necropsy studies, drug eluting stents delayed arterial healing and re-endothelialisation compared with bare metal stents. ${ }^{2324}$ Again, this effect may be enhanced in people with diabetes, particularly in the absence of dual antiplatelet therapy. Moreover, overall longer stents in people with diabetes may result in a prolongation of the healing and re-endothelialisation process. Finally, people with diabetes may be more likely to experience aspirin resistance than people without diabetes ${ }^{25}$ and may have a particular benefit from a complementary antiplatelet therapy with clopidogrel or ticlopidine. Taken together, these mechanisms may explain why prolonged dual antiplatelet therapy may be particularly important in people with diabetes.

We recently reported a potentially reduced average risk of myocardial infarction associated with sirolimus eluting stents compared with bare metal stents but not with paclitaxel eluting stents compared with bare metal stents. ${ }^{1}$ Our analysis suggests that this effect is likely to be present in people with diabetes, but wide credibility intervals of stratified analyses preclude firm conclusions. Stone et al found that about $1.4 \%$ of patients undergoing target lesion revascularisation developed a myocardial infarction associated with the procedure. ${ }^{26}$ A stent that decreases revascularisation rates will therefore result in a reduction of procedure related myocardial infarctions but this will result in a maximum reduction of the relative risk of about $4 \%$, whereas the actually observed average relative risk reduction was $19 \% .{ }^{1}$ Other mechanisms will therefore have to be considered. The process of restenosis in itself may result in myocardial infarction in some patients, ${ }^{27}$ and a more pronounced reduction of restenosis of sirolimus eluting stents may directly contribute to decreasing the risk of myocardial infarction. Although stent thrombosis is uncommon it is associated with a high incidence of myocardial infarction, ${ }^{26}$ and the benefits of drug eluting stents for myocardial infarctions are less likely to be offset by stent thromboses in sirolumus eluting stents than in paxlitaxel eluting stents. ${ }^{1}$

The mechanisms of action of the two drugs merit some consideration. Sirolimus, a macrocyclic lactone, inhibits mammalian target of rapamycin (mTOR) thereby blocking cell division by interfering at the transition from G1 to S phase. ${ }^{28}$ People with diabetes show a breakdown in the phosphatidylinositol 3 kinase insulin signal transduction pathway, where mTOR has an important role, ${ }^{29}$ and this has been thought to limit the effectiveness of sirolimus in people with diabetes mellitus. Paclitaxel, which affects cell replication by stabilising microtubules, does not seem to be influenced by insulin resistance and was therefore hypothesised to be superior to sirolimus in people with diabetes. The present study does not support these speculations: although paclitaxel eluting stents tended to reduce the risk of target lesion revascularisation more strongly in people with diabetes than in people without diabetes, sirolimus eluting stents were superior to paclitaxel eluting stents in reducing the risk of revascularisation in people both with and without diabetes.

Rapid technological developments led to novel, second generation drug eluting stents using different antiproliferative substances, absorbable polymers used as drug carriers, and fully bioabsorbable stent systems. Although the short term results for effectiveness of these new generation devices are encouraging, ${ }^{30-32}$ their long term safety profile in people with and without diabetes is still ill defined. An expansion of the present network meta-analysis will be required as soon as longer term follow-up data become available for these novel stent systems.

\section{Conclusion}

In trials with a duration of dual antiplatelet therapy of six months or longer drug eluting stents were safe and effective in people with and without diabetes. It seems prudent to adhere to a minimal duration of dual antiplatelet therapy of six months in patients undergoing implantation of a drug eluting coronary stent. The potential benefits of a longer duration of therapy need to be balanced against potential risks, such as clinically relevant bleeding. The optimal duration can only be determined in adequately powered large scale randomised controlled trials.

We thank Boston Scientific and Cordis for the provision of additional data. CTU Bern is supported by the Swiss National Science Foundation. Contributors: CSt and SA contributed equally to the manuscript. PJ and CS conceived the study. PJ, CSt, SWa, SA, and SWi were responsible for the conception and design of the study. SWa, CSt, SA, and PJ did the analysis and interpreted the analysis in collaboration with SWi. CSt, SA, AK, MCM AS, MEP, GWS, MBL, JSdL, JJG, SJP, MS, MJS, HK, CSp, MM, PV, MTD, PC, ASP, AJN, PD, BM, SWi, and PJ were responsible for the acquisition of data. PJ, CSt, SA, and SWi wrote the first draft of the manuscript. All authors critically revised the manuscript for important intellectual content and approved the final version of the manuscript. CSt and PJ obtained public funding. PJ, CSt, PD, BM, and SWi provided administrative, technical, and logistic support.

Funding: Swiss National Science Foundation (grant Nos 3233B0-115212, 3233-066377, and 3200-066378) to CSt and PJ. The implementation and validation of the statistical models used for this study were funded by the Swiss National Science Foundation's national research programme 53 (grant No 405340-104762) to PJ. CSt and PJ are PROSPER (program for social medicine, preventive and epidemiological research) fellows funded by the Swiss National Science Foundation. The funder had no role in study design, data collection, data analysis, data interpretation, or writing of the report.

Competing interests: CSt and PJ report receiving unrestricted grants from the Swiss National Science Foundation. AK receives lecture fees from Bristol-Myers Squibb, Cordis, GlaxoSmithKline, Lilly, Medtronic, Novartis, 


\section{WHAT IS ALREADY KNOWN ON THIS TOPIC}

People with diabetes are at an increased risk for coronary heart disease and have more restenoses after the implantation of coronary stents

A meta-analysis suggested a strongly increased risk of death associated with sirolimus eluting stents compared with bare metal stents in people with diabetes

\section{WHAT THIS STUDY ADDS}

Reported increases in the risk of death associated with drug eluting stents compared with bare metal stents in people with diabetes were probably due to dual antiplatelet therapy lasting less than six months in early trials

In trials with dual antiplatelet therapy for six months or longer drug eluting stents were safe and effective in people both with and without diabetes

In clinical practice it seems prudent to adhere to a minimal duration of dual antiplatelet therapy of six months in all patients undergoing implantation of a drug eluting coronary stent

and Sanofi-Aventis. MCM receives lecture fees from Cordis, Boston Scientific, and Abbot, which go to a research organisation (RCF, Massy, France). AS receives unrestricted grant support for the Department of Cardiology he chairs from Amersham/General Electric, Bayerische Forschungsstiftung, Bristol-Myers Squibb, Cordis, Cryocath, Guidant, Medtronic, Nycomed, and Schering. MEP receives lecture fees from Medtronic. GWS receives consulting fees from Boston Scientific, Abbott, Guidant, Xtent, and BMS Imaging, lecture fees from Boston Scientific, Abbott, and Medtronic, has equity interests in Devax and Xtent, and is a member of the board of directors of Devax. MBL receives consulting fees from Cordis, Medtronic, Boston Scientific, and OrbusNeich and has equity interests in Conor, Medinol, and OrbusNeich. JJG is on the advisory board of Boston Scientific and receives research grant support from Cordis. SJP receives research grant support from Cordis. HK receives unrestricted grant support from Cordis. CSp receives consulting and lecture fees from Cordis, Boston Scientific, Abbot, Lilly, and Pfizer. MTD receives lecture fees from Boston Scientific. BM receives research grant support from various stent companies, including Cordis and Boston Scientific, and is in the speaker bureau for various stent companies, including Cordis and Boston Scientific. SW receives lecture and consulting fees from Abbot, Biotronic, Biosensors, Boston Scientific, Cordis, and Medtronic. GWS and MBL are directors of the Cardiovascular Research Foundation, a public charity affiliated with Columbia University Medical Center, from which they receive no compensation; the Cardiovascular Research Foundation receives research or educational funding from Boston Scientific, Cordis, Sanofi-Aventis, and Bristol-Myers Squibb.

Ethical approval: Not required.

Provenance and peer review: Not commissioned; externally peer reviewed.

1 Stettler C, Wandel S, Allemann S, Kastrati A, Morice MC, Schomig A, et al. Outcomes associated with drug-eluting and bare-metal stents: collaborative network meta-analysis. Lancet 2007;370:937-48.

2 Moussa I, Leon MB, Baim DS, O’Neill WW, Popma IJ, Buchbinder M, et al. Impact of sirolimus-eluting stents on outcome in diabetic patients: a SIRIUS (SIRollmUS-coated Bx Velocity balloonexpandable stent in the treatment of patients with de novo coronany artery lesions) substudy. Circulation 2004;109:2273-8.

3 Hermiller IB, Raizner A, Cannon L, Gurbel PA, Kutcher MA, Wong SC, et al. Outcomes with the polymer-based paclitaxel-eluting TAXUS stent in patients with diabetes mellitus: the TAXUS-IV trial. J Am Coll Cardiol 2005;45:1172-9.

4 Abizaid A, Costa MA, Blanchard D, Albertal M, Eltchaninoff $\mathrm{H}$, Guagliumi G, et al. Sirolimus-eluting stents inhibit neointimal hyperplasia in diabetic patients. Insights from the RAVEL trial. Eur Heart/ 2004;25:107-12.

5 Spaulding C, Daemen J, Boersma E, Cutlip DE, Serruys PW. A pooled analysis of data comparing sirolimus-eluting stents with bare-metal stents. N Engl J Med 2007;356:989-97.

6 Kastrati A, Mehilli J, Pache J, Kaiser C, Valgimigli M, Kelbaek H, et al. Analysis of 14 trials comparing sirolimus-eluting stents with baremetal stents. N Engl/ Med 2007;356:1030-9.

7 Lumley T. Network meta-analysis for indirect treatment comparisons. Stat Med 2002:21:2313-24.

8 Psaty BM, Lumley T, Furberg CD, Schellenbaum G, Pahor M, Alderman $\mathrm{MH}$, et al. Health outcomes associated with various antihypertensive therapies used as first-line agents: a network metaanalysis. JAMA 2003;289:2534-44.

9 Lu G, Ades AE. Combination of direct and indirect evidence in mixed treatment comparisons. Stat Med 2004;23:3105-24.

10 Caldwell DM, Ades AE, Higgins IP. Simultaneous comparison of multiple treatments: combining direct and indirect evidence. $B M$ 2005;331:897-900.

11 Cooper NJ, Sutton Al, Lu G, Khunti K. Mixed comparison of stroke prevention treatments in individuals with nonrheumatic atrial fibrillation. Arch Intern Med 2006;166:1269-75.

12 Mauri L, Hsieh WH, Massaro JM, Ho KK, D’Agostino R, Cutlip DE. Stent thrombosis in randomized clinical trials of drug-eluting stents. NEnglJ Med 2007;356:1020-9.

13 Cutlip DE, WindeckerS, Mehran R, Boam A, Cohen DJ, van Es GA et al. Clinical end points in coronary stent trials: a case for standardized definitions. Circulation 2007;115:2344-51.

14 Juni P, Altman DG, Egger M. Systematic reviews in health care: assessing the quality of controlled clinical trials. BMJ 2001;323:42-6.

15 Smith TC, Spiegelhalter DJ, Thomas A. Bayesian approaches to random-effects meta-analysis: a comparative study. Stat Med 1995:14:2685-99.

16 Serruys PW, van Hout B, Bonnier H, Legrand V, Garcia E, Macaya C, et al. Randomised comparison of implantation of heparin-coated stents with balloon angioplasty in selected patients with coronary artery disease (Benestent II). Lancet 1998;352:673-81.

17 Pinto DS, Stone GW, Ellis SG, Cox DA, Hermiller J, O'Shaughnessy C, et al. Impact of routine angiographic follow-up on the clinical benefits of paclitaxel-eluting stents: results from the TAXUS-IV trial. J Am Coll Cardiol 2006;48:32-6.

18 Tu JV, Bowen J, Chiu M, Ko DT, Austin PC, He Y, et al. Effectiveness and safety of drug-eluting stents in Ontario. $N$ Engl J Med 2007;357:1393-402.

19 Pocock SJ. Safety of drug-eluting stents: demystifying network metaanalysis. Lancet 2007;370:2099-100.

20 Thompson SG. Why sources of heterogeneity in meta-analysis should be investigated. BMJ 1994;309:1351-5.

21 Stettler C, Allemann S, Juni P, Cull CA, Holman RR, Egger M, et al. Glycemic control and macrovascular disease in types 1 and 2 diabetes mellitus: meta-analysis of randomized trials. Am Heart 2006;152:27-38.

22 Daemen J, Wenaweser P, Tsuchida K, Abrecht L, Vaina S, Morger C, et al. Early and late coronary stent thrombosis of sirolimus-eluting and paclitaxel-eluting stents in routine clinical practice: data from a large two-institutional cohort study. Lancet 2007;369:667-78.

23 Finn AV, Nakazawa G, Joner M, Kolodgie FD, Mont EK, Gold HK, et al. Vascular responses to drug eluting stents: importance of delayed healing. Arterioscler Thromb Vasc Biol 2007;27:1500-10.

24 Joner M, Finn AV, Farb A, Mont EK, Kolodgie FD, Ladich E, et al. Pathology of drug-eluting stents in humans: delayed healing and late thrombotic risk. I Am Coll Cardiol 2006;48:193-202.

25 Evangelista V, Totani L, Rotondo S, Lorenzet R, Tognoni G, De Berardis $\mathrm{G}$, et al. Prevention of cardiovascular disease in type-2 diabetes: how to improve the clinical efficacy of aspirin. Thromb Haemost 2005;93:8-16.

26 Stone GW, Ellis SG, Colombo A, Dawkins KD, Grube E, Cutlip DE, et al. Offsetting impact of thrombosis and restenosis on the occurrence of death and myocardial infarction after paclitaxel-eluting and bare metal stent implantation. Circulation 2007;115:2842-7.

27 Doyle B, Rihal CS, O'Sullivan Cl, Lennon R], Wiste HJ, Bell M, et al. Outcomes of stent thrombosis and restenosis during extended follow-up of patients treated with bare-metal coronary stents. Circulation 2007;116:2391-8.

28 Gingras AC, Raught B, Sonenberg N. mTOR signaling to translation. Curr Top Microbiol Immunol 2004;279:169-97.

29 Carter Al. TOR of the cell cycle: are there important implications for diabetics in the era of the drug-eluting stent? Catheter Cardiovasc Interv 2004;61:233-6.

30 Ormiston JA, Serruys PW, Regar E, Dudek D, Thuesen L, Webster MW, et al. A bioabsorbable everolimus-eluting coronary stent system for patients with single de-novo coronary artery lesions (ABSORB): a prospective open-label trial. Lancet 2008;371:899-907.

31 Stone GW, Midei M, Newman W, Sanz M, Hermiller JB, Williams J, et al. Comparison of an everolimus-eluting stent and a paclitaxel-eluting stent in patients with coronary artery disease: a randomized trial. JAMA 2008;299:1903-13.

32 Mehta RH, Leon MB, Sketch MH Jr. The relation between clinical features, angiographic findings, and the target lesion revascularization rate in patients receiving the endeavor zotarolimuseluting stent for treatment of native coronary artery disease: an analysis of Endeavor I, Endeavor II, Endeavor II continued access registry, and Endeavor III. Am / Cardiol 2007;100(8B):62M-70M.

Accepted: 27 June 2008 